\title{
Affordable Interactive Virtual Reality system for the Dynamic Hip Screw surgery training in vitro
}

\author{
Amr Ahmed ${ }^{1}$, Mohammad Maqsood ${ }^{2}$, Hassan Saif ${ }^{1}$, Anas Salman ${ }^{1}$ \\ ${ }^{1}$ School of Computer Science, University of Lincoln \\ Lincoln LN6 7TS / UK \\ ${ }^{2}$ United Lincolnshire Hospitals NHS Trust, Lincoln County Hospital, \\ Lincoln, LN2 5QY / UK \\ aahmed@lincoln.ac.uk, Mohammad.Maqsood@ulh.nhs.uk
}

\begin{abstract}
Interactive virtual reality systems provide safe and costeffective training environment to improve the technical skills and competence of surgeons. The trainees can have as many practice sessions, without need to the trainer all the time, before even start carrying out the procedure on any real patient.
\end{abstract}

In this paper, we present an affordable interactive virtual reality system for the Dynamic Hip Screw (DHS) surgery training in vitro, through 3D tracking. The system facilitates a safe (in vitro / off patient) training to improve the cognitive coordination of trainees and junior surgeons, in particular the Hands, Eyes and Brain coordination. The system is based on very cheap commercial off-the-shelf (COT) components, which are very affordable, and needs minimum setup effort and knowledge. It also provides a range of visual and quantitative feedback information and measures, such as position, orientation, insertion point, and depth of drilling. It is envisaged that improving this level of coordination, through the training system, will contribute to reducing the failure rate of the DHS procedure. This means better treatment for patients and less costs for the Health services systems (e.g. UK's NHS system).

\section{KEY WORDS}

Virtual Reality, Simulation, Virtual Training, Tracking, Motion sensors, Computer Vision, DHS, Dynamic Hip Screw.

\section{Introduction}

The fracture of the femur, at the level of the Intertrochenteric area, is a very common injury especially in elderly. These injuries are most commonly treated by the DHS (Dynamic Hip Screw) procedure.

The DHS procedure is most commonly done by junior doctors. However, this procedure is technically demanding, with $4-12 \%$ risk of implant failure, which is a serious complication and may lead to infection, deep vein thrombosis (DVT), and pulmonary embolism (PE). A major reason of the implant failure is the incorrect positioning of the implant at the time of surgery. Hence, the procedure requires high level of Hands, Eyes and Brain coordination.

It is envisaged that improving this level of coordination will contribute to reducing the failure rate of the DHS procedure. This means better treatment for patients, less costs for the NHS. Hence, the importance of this work.

However, there is no affordable system in place to improve the junior doctors' hands, eyes and brain coordination, in vitro, which is crucial for the DHS procedure. Although there are some courses to practice on synthetic bones, those courses are very expensive, approximately $£ 1000$ for the course fee. They also provide very limited practical opportunities for carrying the DHS procedure (almost only one chance to do this procedure on a synthetic bone). This does not give enough chance to practice. Therefore, the trainee has got no other way to practice except on live patients, which leads to further complications due to technical faults of insufficiently trained surgeon.

\section{Related work}

Interactive virtual reality (VR) systems provide safe and cost-effective training environment to improve the technical skills and competence [11]. This was evidenced in the extensive and advanced use of the VR systems in the aviation industry, for training pilots especially in handling rare emergency situations [2]. For the surgical training, the trainees can have as many practice sessions, without need to the trainer all the time, before even start carrying out the procedure on any real patient. Also, serious games provides a good starting point for VR training systems, with its advanced game engines. An example of that is using the development of $3 \mathrm{D}$ serious games for emergency situations and other tasks such as power network maintenance [7]. However, in most cases 
of serious games, the training is more about role-playing and behaviour, rather than focusing on critical cognitive coordination skills that are crucial for surgeons.

The importance and benefit of the simulation in surgery training have been repeatedly emphasized and reported in various reviews [14][6]. The use of virtual reality in training surgeons has been explored for various types of surgical procedures, including neurosurgery [1]. Probably the most relevant systems are the ones directly addressing the Hip surgery training. In [9], a simulation based training system for hip fracture fixation was reported, and aimed to be used in the hospital environment. The main objective of this system is to improve the trainee surgeon's ability to utilise two-dimensional X-rays during the process of implant insertion. The system provides a virtual environment of the operation theatre, with most of the tools. It provides an excellent insight and information on the procedure, correct positions and alignments. However, it does not provide the intuitive interaction to achieve them, through physical training objects. This means that it is different from our work as it does not facilitate for improving the real-time brain, hands, eyes coordination. Similarly the Bonedoc DHS simulator system that is reported in [10] still misses this coordination training aspect, which is very important for the crucial step of inserting the guide-wire for the DHS procedure.

A bit more generic simulation system for orthopaedic surgery is reported in [3] and updated in [8]. The objective is to provide simulation software that can run on PC, laptop, with minimum requirements, which similar to our objective in one sense. But again this system focuses on the realistic $3 \mathrm{D}$ models and their real-time rendering and avoids the interaction and input techniques that may need special hardware. This means that it lacks this particular type of training; i.e. developing the brain, hands, eyes, coordination.

The most relevant surgical simulator has recently been reported in [5]. In this system, various virtual models and views are provided. More importantly, the interaction with the virtual scene is more intuitive and implemented through the use of a haptic device. The haptic device tracks the hand movements in 3D and provides more intuitive interaction, compared to the conventional input/interaction devices. The use of haptic devices has been utilised before in similar procedures. Heng et al. utilised a stylus haptic pen in their intelligent virtual environment for Chinese acupuncture learning and training [12].

However, the physical structure of the haptic devices could be limiting for some actions. Moreover, its cost may not be affordable, especially for individuals and/or small training environments.
Kanehira et. al. [13] developed a virtual training system for acupuncture using magnetic sensors for the $3 \mathrm{D}$ tracking. However, the magnetic sensor is relatively bulky, at least compared to the size and weight of the needle. Moreover, the wires that connect the sensor to the receiver are another limitation, in addition to the cost involved.

Hence, to the best of our knowledge, there is no affordable system in place to improve the junior doctors' hands, eyes and brain coordination, in vitro, which is crucial for the DHS procedure. In contrast to above mentioned systems, our proposed training environment does not use expensive nor complicated tools. Instead, we use very cheap tools to achieve both accuracy and real time interactivity during the training session. And we focus mainly on the drilling and guide-wire insertion as the crucial skills-demanding part of the DHS procedure.

Our system, with its details, is presented in the next section, with results and evaluation in section 4.

\section{Proposed system}

Our aim in this research is to provide an interactive computer-based training system to facilitate a safe (in vitro) training to improve the Hands, Eyes and Brain coordination of trainees and junior surgeons. The system is required to satisfy the following:

- The system should provide a real-time interaction; through accurate tracking of the training tool in hand (e.g. drill).

- It is important to design the system so that it is based on cheap commercial off-shelf components (COT), which are affordable.

- The system should need only minimum (close to none) setup effort and knowledge.

- The system is aimed to be suitable, not only for teaching/educational institutions/hospitals, but also for individual trainees to acquire their own training system, hence the above constraints on costs and setup.

- The system should provide a range of visual and quantitative feedback information and measures, such as position, orientation, insertion point, and depth of drilling.

Figure 1 presents a screenshot of our designed system. On the screen, the main view is a perspective view showing the virtual bone to be operated on, with a virtual drill and guide-wire (attached to the front of the drill). In the topleft corner, the two common views (AP and Lateral) are displayed and continuously updated in real-time. The blue-ish bar on the right contains measurements, including position and orientation of the guide-wire/drill and the drilled depth, as well as controls for transparency and various viewing controls. 


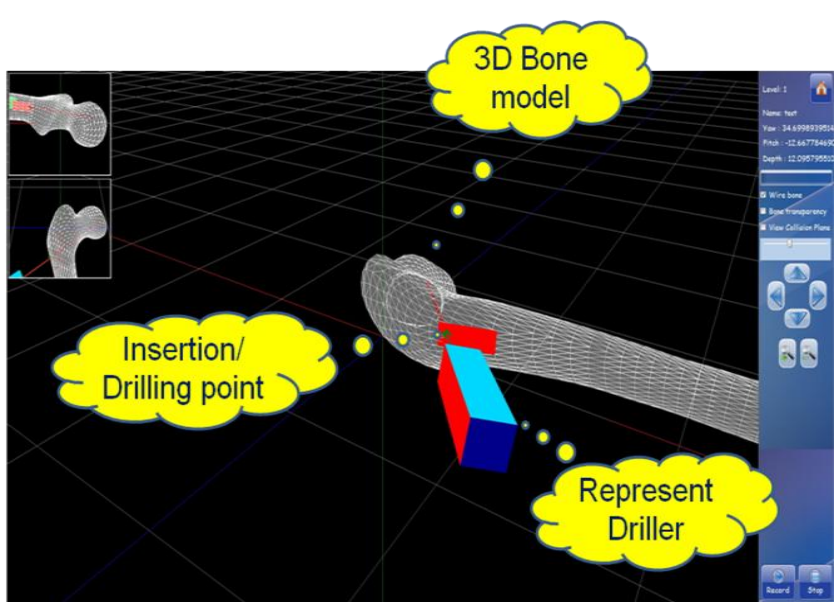

Figure 1: Screenshot of our designed system's screen, showing the virtual bone, various views, feedback, measurements and controls.

In the rest of this section, we present two developed prototypes addressing the above, especially the real-time tracking. The first prototype utilises a combination of motion sensors and computer vision. The second prototype is vision-based with enhanced performance and accuracy.

\subsection{Prototype 1: Combined Motion-sensor and vision}

In this section we explain our first prototype, which implements the tracking of the training tool through the utilisation of motion-sensor (WiiMote + Wii Motion Plus) and vision (webcam). The overall components of the prototype are illustrated in figure 2.

The rotational three degrees of freedom are obtained by analysing the data reported from the accelerometer and the gyroscopes, included in the WiiMote and its associated Wii Motion Plus respectively. So:

$$
\begin{gathered}
\operatorname{roll}=\arctan 2\left(\mathrm{a}_{\mathrm{x}}, \operatorname{sqrt}\left(\mathrm{a}_{\mathrm{y}}{ }^{2}+\mathrm{a}_{\mathrm{z}}{ }^{2}\right)\right) \\
\operatorname{pitch}=\arctan 2\left(\mathrm{a}_{\mathrm{y}}, \operatorname{sqrt}\left(\mathrm{a}_{\mathrm{x}}{ }^{2}+\mathrm{a}_{\mathrm{z}}{ }^{2}\right)\right)
\end{gathered}
$$

In combination with the Wii motion plus we can get yaw. Wii motion plus measure the rate of rotation along all 3axes. So:

$$
\Theta_{\mathrm{i}}=\Theta_{\mathrm{i}-1}+\mathrm{w}_{\mathrm{i}} *\left(\mathrm{t}_{\mathrm{i}}-\mathrm{t}_{\mathrm{i}-1}\right)
$$

(Where $\mathrm{w}$ is rotational velocity, $\Theta$ is an angle, $\mathrm{t}$ is time, $\mathrm{i}$ is the current data point, $\mathrm{i}-1$ is the previous data point).

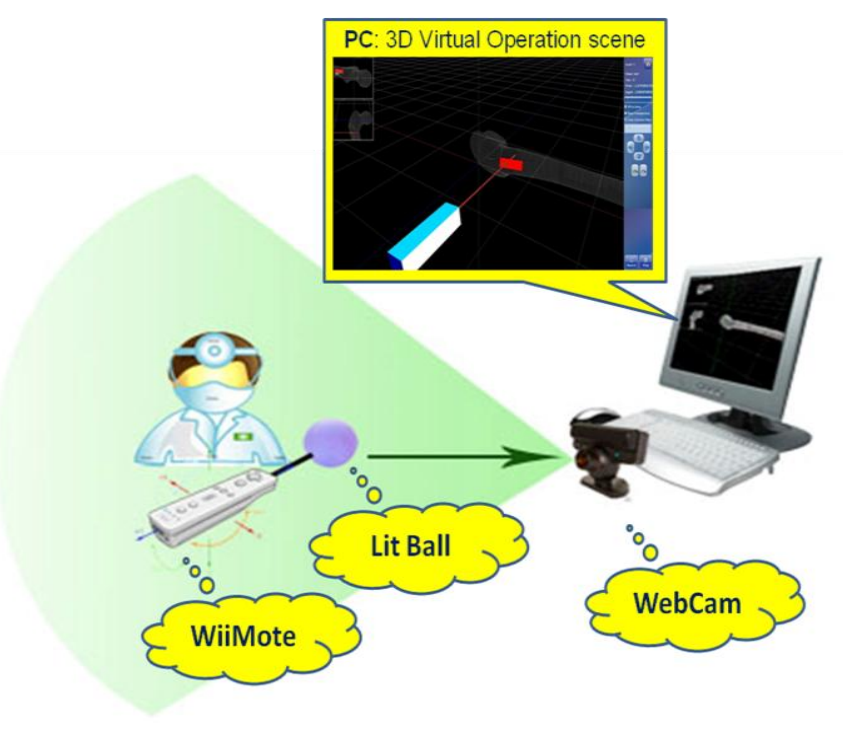

Figure 2: The main components of Prototype 1

Unfortunately, accelerometers become less sensitive to position when they are turned away from perpendicular to gravity. For this reason, the $\mathrm{z}$-axis accelerometer is not very helpful for position sensing when the remote is held in the standard position. Hence, we added the vision element.

For the position, we utilise a lit tennis ball attached to the front of the WiiMote and observed by a webcam, as depicted in figure 3. The ball, projected as a circle in the image, is detected and its area is calculated. From that the radius ' $r$ ' of the projected ball is calculated. Then, given the diameter ' $\mathrm{D}$ ' of the real ball and the focal length ' $\mathrm{f}$ ' of the camera used, the distance ' $\mathrm{L}$ ' from the camera is obtained as follows:

$$
\mathrm{L}=(\mathrm{D} * \mathrm{f}) / 2 * \mathrm{r}
$$

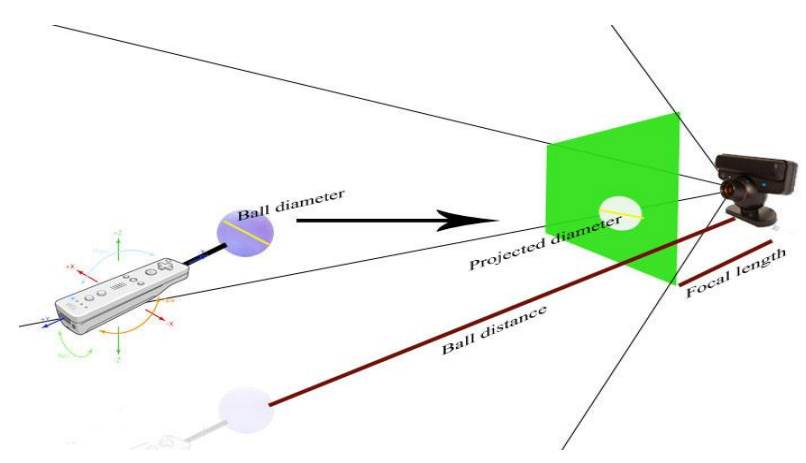

Figure 3: Illustration of depth calculation

We also implemented collision detection, using Ray-Plane intersection detection.

In our implementation of this prototype, we found that, the full tracking of the Motion Plus is good only for a 
limited time period; few seconds. That's because the accumulation of errors from integration over the time. So we need to calibrate the application from time to time. Hence, we looked for enhancement, as discussed in the Prototype 2.

\subsection{Prototype 2: Vision-based}

Prototype 2 is based on vision only. We attach to the drill a set of four coloured markers, in a specific special arrangements, see figure 4 . This markers arrangement facilitates for calculating the six degrees of freedom by detecting the $2 \mathrm{D}$ position of the four coloured markers in the image captured by the camera.
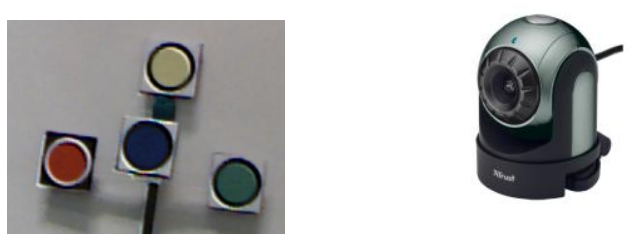

Figure 4: The main components of the visionbased interaction in Prototype 2; The set of four coloured markers and the camera.

The system consists of the four phases, as depicted in figure 5. Those phases are discussed below:

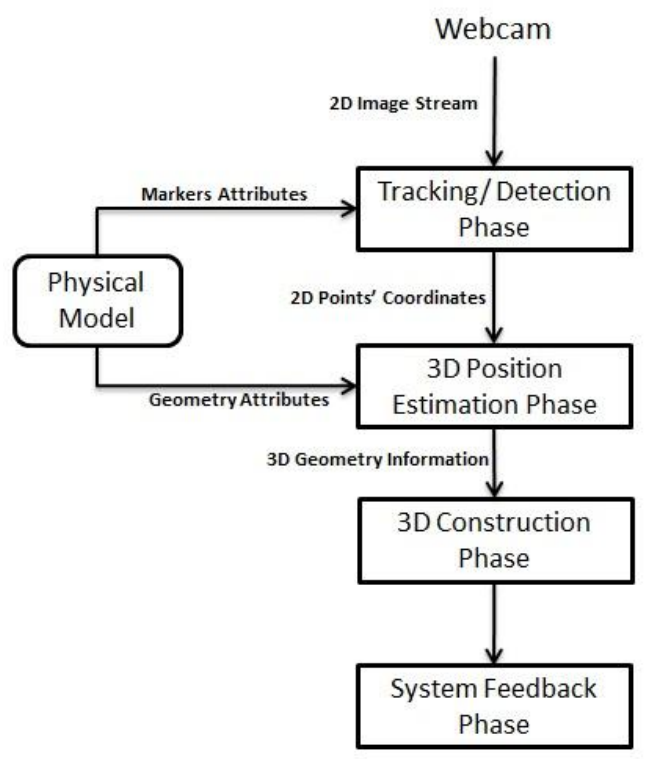

Detection Phase: Using image stream provided by the webcam, the system detects the $2 \mathrm{D}$ position of all coloured markers that are attached to the training drill/tool. To simplify the tracking process we chose a circular shape for all markers. Hough transform was used to detect the four circular markers. Using HSV colour scheme we calculated the Hue histogram to determine the colour of each marker. Algorithm 1 describes the detection process as follows:

Algorithm 1. (Markers' 2D Position Extraction)

Input: 2D image

Output: 2D position for each marker

1: Convert input image to greyscale image

2: Smooth the greyscale image, using Gaussian filter

3: Detect circles using the Fast Hough Transform

4: Remove noisy circles (not matching predefined specific radius' values)

5: for each detected circle do

6: convert image area containing this circle to HSV colour scheme

7: find Hue histogram for the circle's area

8: take the highest colour value from the histogram

9: if the colour value is one of the predefined colour values then

10: return the position of this circle (2D Position of its center)

11: end if

12: end for

The results of testing Algorithm 1 showed very good results in determining the marker's colour in several different lighting conditions. This is demonstrated in figures 6 and 7. Figure 6 shows the histogram of the "Green" marker, before applying the above algorithm. This clearly showing that the green is not dominant enough and the marker can be missed. The histogram shown in figure 7 is for the same "Green" marker but after applying the above algorithm. One can easily notice that the green is highly dominant and the marker should be detected correctly. This example shows the effectiveness of this algorithm.

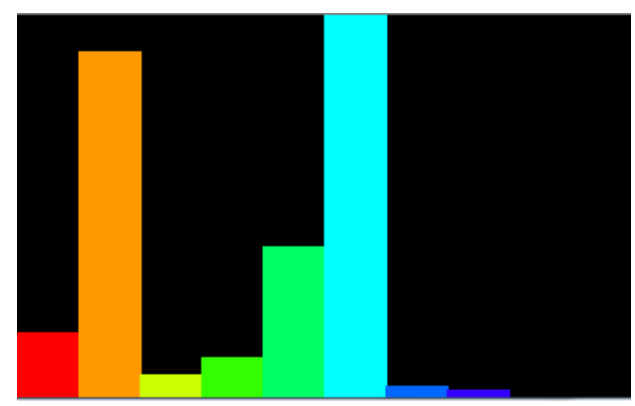

Figure 6: Histogram of a "Green" marker (without applying Algorithm 1). 


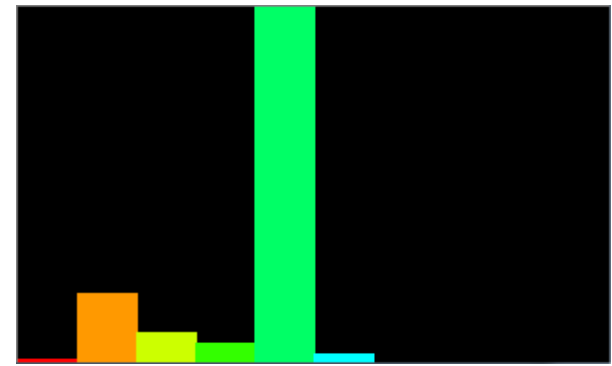

Figure 7: Histogram of a "Green” marker (After Algorithm 1).

3D Posture Estimation Phase: Using both physical object attributes - markers' dimensions and their spatial relative positions - and the determined $2 \mathrm{D}$ coordinates of the four markers, we are able to estimate the $3 \mathrm{D}$ position and orientation of the training object using the POSIT algorithm [4].

The POSIT algorithm ("Pose from Orthography and Scaling with Iteration") is used to extract the 3D position and orientation (6DOF) for an object with pre-known physical dimensions. To extract this 3D information we need the 2D corresponding position for our four noncoplanar markers which we obtained from the previous Detection phase.

The first part of the algorithm, pose from orthography and scaling (POS), assumes that all points on the object (markers set) are all at effectively the same depth and that the markers set are behaving as a rigid body; i.e. any size variations (from the original predefined model) are due solely to the scaling produced by changing the distance from the camera. In this case there is a closed-form solution for that object's 3D pose based on scaling. The assumption that the object points are all at the same depth effectively means that the object is far enough away from the camera that we can neglect any internal depth differences within the object; this assumption is known as the weak-perspective approximation.

Given that we know the camera intrinsic parameters, we can find the perspective scaling of our known object and thus compute its approximate pose. This computation will not be very accurate, but we can then project where our four observed points would go if the true $3 \mathrm{D}$ object were at the pose we calculated through POS. We then start all over again with these new point positions as the inputs to the POS algorithm. This process typically converges within four or five iterations to the true object posehence the name "POS algorithm with iteration" (POSIT).

\section{Results and Evaluation}

The system provides a range of visual and quantitative feedback information and measures, such as position, orientation, insertion point, and depth of drilling. It also allows the trainee/tutor to control the transparency of the bone for training purposes. Moreover, the system provides the same views (AP and Lateral views) as it is the case in the real DHS procedure, which increasing the trainee's experience and linking it with the real procedure. All those facilities are highlighted in figure 8 , and have been recommended and appreciated by Orthopaedic consultants.

Test results showed that the POSIT algorithm produces high accuracy in determining the $3 \mathrm{D}$ position and orientation of the tracked training tool (e.g. drill). Quantitative evaluation is also summarised in Table 1.

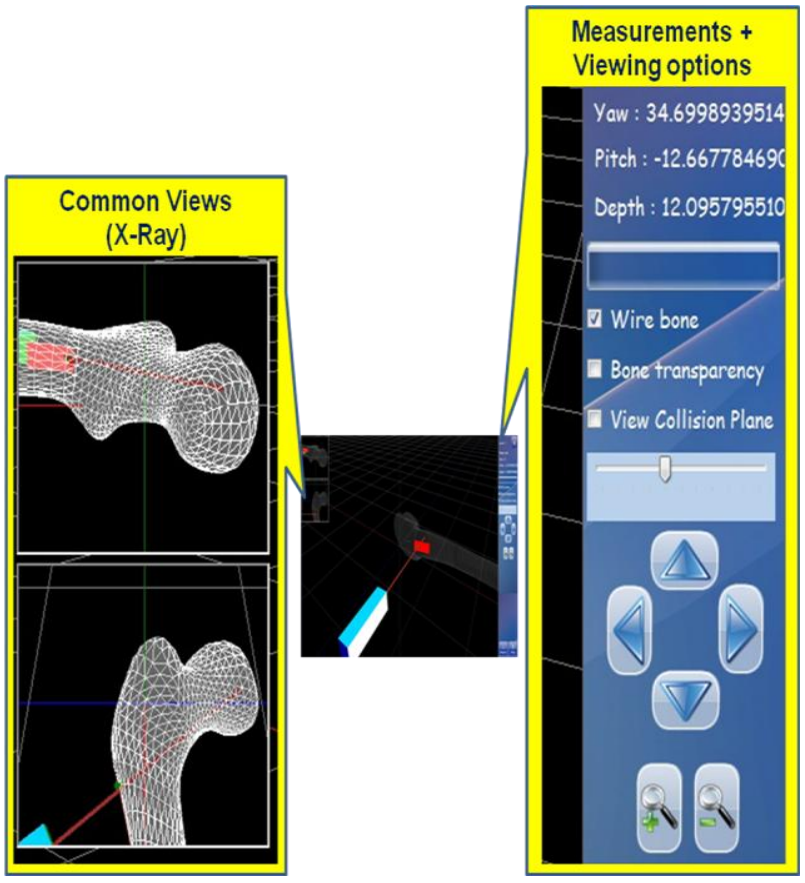

Figure 8: The system's facilities highlighted

\begin{tabular}{|c|c|}
\hline Criteria & Value \\
\hline $\begin{array}{l}\text { Accuracy of } \\
\text { Dimensions }\end{array}$ & $95 \%$ \\
\hline Markers Type & Circles \\
\hline $\begin{array}{l}\text { Color Variance } \\
\text { Background) }\end{array}$ & $100 \%$ \\
\hline Circles radius size & $0.7 \mathrm{~cm}$ \\
\hline Screen Resolution & $640 \times 480$ \\
\hline $\begin{array}{l}\text { Object Dimensions (width, height, } \\
\text { depth) }\end{array}$ & $(7,5,2) \mathrm{cm}$ \\
\hline Translation Error $(\mathbf{X}, \mathbf{Y}, \mathbf{Z})$ & $(0.05,0.1,0.2) \mathrm{cm}$ \\
\hline Rotation Error (rx, ry, rz) & $(0.3,0.2,0.5)$ degree \\
\hline Tracking Accuracy & $97 \%$ \\
\hline $\begin{array}{l}\text { Maximum distance from the } \\
\text { camera }\end{array}$ & $42.3 \mathrm{~cm}$ \\
\hline Minimum distance to the camera & $8.4 \mathrm{~cm}$ \\
\hline Rotation Freedom & $(30,30,360)$ \\
\hline
\end{tabular}

Table 1: Summary of quantitative evaluation of the system's accuracy. 


\section{Conclusion}

We presented an affordable computer-based interactive virtual reality training system, for the DHS procedure in vitro. The training system facilitate a safe (in vitro / off patient) training to improve the Hand, Eyes and Brain coordination of trainees and junior surgeons. This is important for surgeons to be able to place the guide-wire in the perfect desired position in the first trial. The key element in facilitating the evaluation and development of this coordination is the 3D tracking, which is the main emphasis of our extermination.

The system is based on Commercial Off-The-shelf (COT) components, which are cheap and affordable, and needs minimum setup effort and knowledge. This makes it suitable, not only for teaching/educational institutions/hospitals, but also for individual trainees to acquire their own training system.

The system provides a range of visual and quantitative feedback information and measures, such as position, orientation, insertion point, and depth of drilling. It also allows the trainee/tutor to control the transparency of the bone for training purposes. Moreover, the system provides the AP and Lateral views as it is the case in the real DHS procedure, which increasing the trainee's experience and linking it with the real procedure.

It is recognised that the system lacks the force feedback to trainees, compared with using haptic devices. However, for placing the guide-wire, simple feedback (resistance) can be added to the current system. The system is also flexible enough to register and align the virtual bone with physical locations as a reference. The rotation angle ranges may seem to be limited. However, they were found satisfactory for the specific task in the guide-wire insertion of the DHS, which is the main emphasis.

It is believed that trainees who have developed the coordination using this device can place the guide wire in ideal position in the first attempt. It is envisaged that improving this level of coordination will contribute to reducing the failure rate of the DHS procedure. This means better treatment for patients and less costs for the Health Services.

\section{References}

[1] A. Alaraj, M. Lemole, J. Finkle, R. Yudkowsky, A. Wallace, C. Luciano, P. Banerjee, S. Rizzi, F. Charbel, Virtual reality training in neurosurgery: Review of current status and future applications, Surgical Neurology International, 2011, 2(1), 2-52.

[2] A. Mohan, M. Proctor, Virtual Reality - A 'play station' of the future, A review of virtual reality and orthopaedics. Journal of Acta Orthopaedica Belgica, 72(6), 2006, 659-663.

[3] A. Sourin, O. Sourina, T. Howe, Virtual orthopaedic surgery training. IEEE Computer Graphics and Applications, 20(3), 2000, 6-9.

[4] D. Dementhon, L. Davis, Model-based object pose in 25 lines of code. International Journal of Computer Vision, 1995, 15(1-2), 123-141.

[5] J. Froelich, J. Milbrandt, W. Novicoff, K. Saleh, D. Allan, Surgical Simulators and Hip Fractures: A Role in Residency Training?. Journal of Surgical Education, 68(4), 2011, 298-302.

[6] L. Sutherland, P. Middleton, A. Anthony, J. Hamdorf, P. Cregan, D. Scott, G. Maddern, Surgical simulation: a systematic review, Annals of Surgery, 243(3), 2006, 291-300.

[7] M. Rosendo, T. Buriol, K. de Geus, S. Scheer, C. Felsky, Towards the Development of a 3D Serious Game for Training in Power Network Maintenance, In proceeding of Games and Virtual Worlds for Serious Applications, 2011, 16-23.

[8] O. Sourina, Orthopedic surgery training simulation. Journal of Mechanics in Medicine and Biology, 7(1), 2007, 37-53.

[9] P. Blyth, N. Stott, I. Anderson, A simulation-based training system for hip fracture fixation for use within the hospital environment. INJURY, 38(10), 2007, 1197-1203.

[10] P. Blyth, P. Sehgal, Use of the Bonedoc DHS simulator by fifth year medical students: A pilot study. In proceedings of the ascilite conference, Auckland, 2009, 74-80.

[11] P. Heng, C. Cheng, T Wong, Virtual Reality Techniques - Application to anatomic visualisation and Orthopaedic training. Clinical Orthopaedics, 442, 2006, 5-12.

[12] P. Heng, T. Wong, R. Yang, Y. Chui, Y. Xie, K. Leung, P. Leung, Intelligent Inferencing and Haptic Simulation for Chinese Acupuncture Learning and Training. IEEE TRANSACTIONS ON INFORMATION TECHNOLOGY IN BIOMEDICINE, 10(1), 2006, 28-41.

[13] R. Kanehira, A. Shoda, M. Yagihashi, H. Narita, H. Fujimoto, Development of an Acupuncture Training System Using Virtual Reality Technology, In proceeding of the Fuzzy Systems and Knowledge Discovery, 2008, $665-668$.

[14] S. Tan, S. Sarker, Simulation in surgery: a review, Scottish Medical Journal, 56(2), 2011, 104-109. 